\title{
Reflections and Forethoughts on Global Clinical and Translational Research
}

\author{
Claude Hughes, Fengyu Zhang
}

December 24, 2020

DOI:10.36316/gcat.02.0038

\section{Reflections on Global Clinical and Translational Research}

By releasing the final issue of 2020, the journal Global Clinical and Translational Research has attained its second anniversary. We want to take this opportunity to reflect upon our successes to date and look forward and think about the journal's foreseeable future and the broad range of scientific research and innovations that may grace our pages in the future.

While all of us experienced the global pandemic of coronavirus disease-19 (COVID-19) in 2020 with its severe attendant interruption of regular economic activities and daily life, including our research and teaching work, numerous scientists shifted their research focus in order to participate in the global effort to respond in a multitude of ways. While a few scientists and physicians have been highly effective in communicating essential facts and changing scientific insights to the general public, many others in many disciplines in nations worldwide have been diligently working to produce data that truthfully updates our evolving understanding of COVID-19. That broad spectrum of largely underappreciated work has and does continue to contribute to meeting the needs of this global public health emergency. A non-exhaustive but illustrative set of disciplines ranges from epidemiology (including counting daily cases and death reported worldwide), clinical care and pathological reporting, vaccine development, and clinical trials to test existing drugs that might be repurposed for preventing and treating COVID-19 patients. Like many of those colleagues who have added COVID-19 to their research portfolio along with their long-standing previous core research themes, our new journal has managed to retain our broad scope of presenting papers on important topics in various areas of clinical and translational research and incorporate aspects of COVID-19 as a part of our two years of accomplishments and progress. We will now reflect and summarize some of those themes that have appeared in Global Clinical and Translational Research and then look forward to some topics that we hope to address in the next few years of the journal's life.

A summary of publications related to the journal's initial commitments

We have published nearly 40 items in the past two volumes, including research articles, reviews, survey reports, commentaries, and editorials. These publications are mainly grouped into clinical and translational research from three dimensions in genomics, environmental, and social aspects of human disease and health. Here we review the past publications and provide our perspectives.

Clinical research. Jiang et al. reported a case of ultralow rectal cancer that after treatment with firing-needle acupuncture and chemotherapy, the tumor completely disappeared. Liu et al. reported that the levels of serum oxidants and protein S100B are associated in the firstepisodes and drug-naïve schizophrenia patients. Wang et al. reported an antipsychotic treatment study in parallel group of patients with the first-episode drugnaïve and medication-free acute phase. With retrospecttive analysis, Zhang et al. reported the management and treatment of pregnant women with pulmonary arterial hypertension associated with congenital heart disease, and Peng et al. published a case report on prevention of hypertriglyceridemia-induced pancreatitis in a pregnant woman. In addition, to address the COVID-19 pandemic, Zhang and Hughes published a systematic review of the clinical epidemiology of COVID-19, followed by another review of the clinical characteristics by Qiu and colleagues.

Genomic research. Xia et al. reported a large-scale combined genome-wide association analysis of five major neuropsychiatric disorders and identified genetic variants shared across disorders, which provides valuable information about several human diseases' genetics. Zhang and Walters published a review of viral genomics and host cellular susceptibility factors in COVID-19. This review is the first article that systematically describes the genomic structure and proteome of severe acute respiratory syndrome virus-2 (SARS-CoV-2) that causes the disease and host receptors and proteases reported to influence viral entry to the host cell that is required for establishing an infection. This article provides important structured insights into the etiology and viral-host interactions in COVID-19 and the implications for the future development of strategies for early interventions.

Environmentally-related human diseases and health research. Hughes and Adibe published a review of gastroschisis risk factors, possible mechanisms, and potential mitigative interventions. Following this, Liu et al. reported an ecological analysis of cross-sectional and 
time-series data related to the occurrence of gastroschisis. They showed that reduced job participation in heavy manufacturing was significantly associated with the decline of gastroschisis in 14 cities of Liaoning, China, from 2006 to 2015 . This finding is in contrast to the conclusions drawn from some previous studies in the US. $\mathrm{Ou}$ et al. reviewed genes and environmental factors related to autism spectrum disorder and published a research article on prenatal and perinatal factors associated with autism spectrum disorders.

Microbiome and exposome research. There is now a broad acceptance that human microbiomes are important microenvironmental elements of the bodily interface with the exogenous environment. The gut microbiome has drawn significant attention, particularly the concept of a gut-brain axis and potential relationships with neuropsychiatric disorders. Li et al. reviewed the gut microbiome and autism by discussing the potential mechanistic and therapeutic implications. Kang et al. reviewed the gut microbiome and antipsychotic-induced metabolic alterations in patients with schizophrenia. Zhang and Mattison published an editorial on measuring the exposome associated with health, which was partially commentary on an article "The Exposome and Health: Where Chemistry Meets Biology" that had been published in Science (2). They discussed social and epidemiological surveys as complementary approaches to better understand the relationship between the exposome and health. In an additional article that was based on a national representative longitudinal social survey, Li and Lu reported that early childhood adversity was significantly associated with depression in later life, providing one illustration that social survey may reveal some historic events and their impact.

Social environment and health research. One of the themes that the editorial board members have supported is the importance of social factors as important environmental influences on human health and disease. Clinical and translational research is ultimately intended to improve the health and longevity of human individuals. The social environment is a potent determinant of healthful longevity and may undermine or enhance individuals' ability to live with various chronic diseases. Based on a national-representative survey, Xia and Li published a report that socioeconomic status affects hypertension risk of older adults in China.

In contrast, Obasanjo and Mann published a report that examined the employment status and management of type 2 diabetes. Also, Ye et al. published a qualitative study of cervical cancer survivors using a focus group approach to explore the factors associated with qualityof-life. Zhang and Zhang published an article about social networks and functional health status among the elderly. Furthermore, Ren et al. published an article that examined occupation and family burden on psychological adjustments among returning migrants based on a survey conducted in multiple regions in China.

Lastly, we offered some comments about the broad issues of reliability, reproducibility, and ultimately interpretability in scientific research and reporting. We chose to address a) study rigor as a significant challenge in the conduct of human clinical and translational research, acknowledging that various sources of hetero- geneity are hard to observe and control; and b) adding our perspectives regarding the use of the $p$-value to the numerous recent discussions in the scientific literature regarding this issue.

\section{A summary of publications related to the journal's inclusion of research on COVID-19}

The pandemic of COVID-19 has been unprecedented in recent human history. Its profound global impact has not only been a human health catastrophe but also a devastation of other human economic and social activities. The history of this era will eventually be perceived and described in retrospect, but our "real time" assessment is that despite some rare exceptions, a) medical professionals of many sorts have indeed been heroically committed in responding to this disease in the care of overwhelming numbers of ofttimes critically-ill patients; and b) scientific investigators of many sorts have also heroically committed themselves to the research and development work needed to understand this viral pathogen, disease pathogenesis, screening and testing the potential to repurpose existing drugs, and conducting a wide range of clinical trials in patients and healthy volunteers to determine efficacy and safety of numerous potential therapeutics. At the time of this writing, some optimism seems justified as a few vaccines are beginning to be available for administration worldwide. We are unsure how long or how well vaccineinduced immunity may or may not protect against clinical disease as inevitable viral mutations occur.

Continuing research will be needed to discover and develop new drugs to treat or prevent COVID-19 in individuals and populations long into the future. We wish to take this opportunity to highlight just a few of the areas where sustained or expanded research will be needed.

First, treating complications. It is known that about $20 \%$ of clinically symptomatic patients have severe COVID-19. People who have survived severe COVID-19 may have persistent functional deficits due to diverse severe complications during their acute illness. These patients typically experienced cytokine storm and/or multiorgan involvement, including pulmonary, cardiovascular, renal (2), and neurological (3) systems. Therefore, a rising incidence of chronic or recurrent medical issues related to past COVID-19 infections should be expected.

Second, post-exposure prophylaxis drugs. While there is no information yet available regarding the durability of protection by vaccination-based immunization, it seems unlikely that effective immunity will be life-long for much of the global human population. Therefore, along with periodic/continuing development of updated vaccines to approximate the evolution of SARS-CoV-2 viral strains and mutations, it will be necessary to continue discovering and developing safe and effective drugs for post-exposure prophylaxis or preemptive therapy to reduce or mitigate disease development once infected. While experience has already shown that a plausible repurposed drug may be inefficacious or worse (4-6), the effort to seek others that may be safe and efficacious should still be made (7).

Lastly, most people have come to realize that a massive problem is that pandemic diseases cause emotional 
trauma and adverse psychological impacts (8). As of December 15, 2020, in search of the terms "COVID-19" and "psychological effects," there have been 1118 articles published or collected in PubMed.gov. These psychological impacts may occur more often in those people who are more susceptible to stress or with some other extreme situations. To date, studies have been conducted on children and students, pregnant women (9), older adults with chronic diseases (10), those who were detained in other countries and suffering financial loss (11), and those who have experienced quarantine (12, 13). The questions are, what is the potential longer-term impact, and what interventions may be safe and effective in these diverse subpopulations?

\section{Forethoughts on Global Clinical and Translational Research}

As we turn our thoughts toward the future and the future of this journal, we hope to receive submissions of manuscripts regarding the noted areas of need in COVID-19 and other areas. For example, along with established epidemiological, public health, clinical trials, and laboratory research, artificial intelligence $(\mathrm{AI})$ applications to any number of "big data" resources should enable enhanced clinical decision-making and preventive health care/medicine. This journal would welcome manuscripts that utilize and even merge those various research approaches in many biomedical research areas, particularly in prenatal therapeutics, translational toxicology, and preventive medicine, including primal, primordial, primary, secondary, tertiary, and quaternary prevention methods. Any ideas for short reviews on potential mechanisms or pathogenic pathways, as well as potential drug targets or other therapeutic strategies focused on these diverse areas, are welcomed.

Finally, we thank our editorial board members and peerreviewers (see acknowledgment of reviewers) who have generously helped us assure the quality of publications and communication of new information and insights to our scientific colleagues with minimal errors.

Editors

Claude L Hughes, MD, PhD

Fengyu Zhang, MSMed, Ph.D., MS

\section{Conflict of Interest}

The authors declare no conflict of interest regarding the publication of this article.

\section{References}

1. Vermeulen R, Schymanski EL, Barabási A-L, Miller GW.
The exposome and health: Where chemistry meets biology. Science. 2020;367(6476):392-6.

2. Shaikh S, Matzumura Umemoto G, Vijayan A. Management of Acute Kidney Injury in Coronavirus Disease 2019. Adv Chronic Kidney Dis. 2020;27(5):377-82.

3. Taherifard E, Taherifard E. Neurological complications of COVID-19: a systematic review. Neurol Res. 2020;42(11): 905-12.

4. Boulware DR, Pullen MF, Bangdiwala AS, Pastick KA, Lofgren SM, Okafor EC, et al. A Randomized Trial of Hydroxychloroquine as Postexposure Prophylaxis for Covid-19. N Engl J Med. 2020;383(6):517-25.

5. Lother SA, Abassi M, Agostinis A, Bangdiwala AS, Cheng MP, Drobot G, et al. Post-exposure prophylaxis or preemptive therapy for severe acute respiratory syndrome coronavirus 2 (SARS-CoV-2): study protocol for a pragmatic randomized-controlled trial. Can J Anaesth. 2020; 67(9):1201-11.

6. Skipper CP, Pastick KA, Engen NW, Bangdiwala AS, Abassi $M$, Lofgren SM, et al. Hydroxychloroquine in Non-hospitalized Adults With Early COVID-19 : A Randomized Trial. Ann Intern Med. 2020;173(8):623-31.

7. Croom E, Zhang F, Hughes C. COVID-19 Interventional and Therapeutic Clinical Trials: Small Molecules, Interactions, Outcomes and Opportunities. Chemistry World [RSC Books, Preprint]. 2020;15 October 2020 (accessed: 15 December 2020). Available from: https://www.chemistryworld.com/reading-room/covid19-interventional-and-therapeutic-clinicaltrials/4012601.article.

8. Holmes EA, O'Connor RC, Perry VH, Tracey I, Wessely S, Arseneault L, et al. Multidisciplinary research priorities for the COVID-19 pandemic: a call for action for mental health science. Lancet Psychiatry. 2020;7(6):547-60.

9. Saccone G, Florio A, Aiello F, Venturella R, De Angelis MC, Locci M, et al. Psychological impact of coronavirus disease 2019 in pregnant women. Am J Obstet Gynecol. 2020;223 (2):293-5.

10. Consonni M, Telesca A, Grazzi L, Cazzato D, Lauria G. Life with chronic pain during COVID-19 lockdown: the case of patients with small fibre neuropathy and chronic migraine. Neurol Sci. 2020.

11. Crayne MP. The traumatic impact of job loss and job search in the aftermath of COVID-19. Psychol Trauma. 2020;12(S1):S180-s2.

12. Brooks SK, Webster RK, Smith LE, Woodland L, Wessely S, Greenberg N, et al. The psychological impact of quarantine and how to reduce it: rapid review of the evidence. Lancet. 2020;395(10227):912-20.

13. Parodi IC, Poeta MG, Assini A, Schirinzi E, Del Sette P. Impact of quarantine due to COVID infection on migraine: a survey in Genova, Italy. Neurol Sci. 2020;41(8):2025-7.

Copyright@ 2020 by the author(s). Licensee Global Clinical and Translational Research. This is an open-access article distributed under the terms and conditions of the Creative Commons Attribution License (CCBY4.0, https://creativecommons.org/licenses/by/4.0/), which permits unrestricted use, distribution, and reproduction in any medium provided the original work is properly cited.

\section{How to cite this article:}

Hughes C and Zhang F. Reflections and Forethoughts on Global Clinical and Translational Research. Glob Clin Transl Res. 2020; 2 (4): 126-128. DOI:10.36316/gcatr.02.0038. 


\section{Acknowledgment of Reviewers}

Global Clinical and Translational acknowledges the following individuals who served as peel reviewers from May 2019 to December 2020. Peer review for some articles published in the first issue was conducted by the Hapres Publisher in UK, so they were not listed here.
R. Donato
J. $\quad \mathrm{Ou}$
A. Grandis
C. Quan
A. Hendrix
Q. Ren*
S. $\mathrm{Hu}$
A. Stefanović
J. Ji
S. Warren
T. $\mathrm{Li}^{*}$
L. $\mathrm{Wu}^{*}$
Y. $\mathrm{Li}$
S. $\mathrm{Wu}$
K. Li
Z. Xu
J. Lu
F. Zhang*
R. Marcantonio
H. Zheng
D. Mattison
Z. Zheng
M. Miljkovic
Y. Zhou
I. Obasanjo*
H. Zhou

Note, ${ }^{*}$, people who served as reviewers for more than one articles published. 